\title{
Cycle threshold responses in SARS-COV2 PCR tests depend on the method by which the samples were obtained and require strict global standardization
}

\author{
Shimon Edelstein ${ }^{1}$ (D) Miriam Sudri ${ }^{1} \cdot$ Shibli Tanous $^{1} \cdot$ Hila Ben Amram $^{2} \cdot$ Adi Sharabi-Nov $^{3} \cdot$ Inna Rozenfeld $^{4}$. \\ Hedva Halal ${ }^{4}$. Salman Zarka ${ }^{5}$
}

Received: 11 January 2022 / Accepted: 1 February 2022 / Published online: 17 February 2022

(c) The Author(s), under exclusive licence to Springer-Verlag GmbH Germany 2022

\begin{abstract}
Purpose Since 2020, a SARS-COV2 epidemic has been raging worldwide. The cycle of the PCR test in which the virus is detected is called cycle threshold (CT). The method of obtaining the sample is not detailed in any published study and is based on general guidelines of the CDC. Our contention is that the manner in which the sample is obtained has a dramatic effect on CT values.

Methods For each person suspected of having Covid-19 who arrives at the emergency room, two swabs are taken in succession, one according to CDC guidelines and the other according to "Ziv" guidelines. The Ziv method sample collection guidelines determine the depth of penetration, the number of rotations of the swab, and their direction. Each double sample was sent for analysis.

Results Analysis of the CT results of the sample to results methods and of the Seegene platform clearly found $(p=0.003$ and $p=0.001$, respectively) that more rigorous sample collection yielded lower CT values.

Conclusion The method of obtaining the samples had a dramatic effect on CT results. Any publication that includes CT results, and certainly studies that discuss CT kinetics, must describe in detail the method by which the samples were obtained. In places where it is also important to detect the onset of illness (airports, hospitals, schools, etc.), it is important to use the Ziv method to reduce the risk of false negatives.
\end{abstract}

Keywords Sars-COV2 $\cdot$ PCR $\cdot$ Cycle threshold $\cdot$ Viral load

To date, the epidemic of COVID-19 has claimed the lives of more than 5,000,000 people [1], with more than 250 million verified cases worldwide. The coronavirus is a respiratory pathogen that binds to epithelial cells in the mucous membranes of the airways, multiplies there, and is secreted in massive amounts from the membranes. One of the ways to

Shimon Edelstein

shimone@ziv.gov.il

1 Infectious Diseases Unit, Ziv Medical Center and Tel-Hai Academic Collage, Tel-Hai, Israel

2 Clinical Microbiology Laboratory, Ziv Medical Center and Tel-Hai Academic Collage, Tel-Hai, Israel

3 Statistical Unit, Ziv Medical Center and Tel-Hai Academic Collage, Tel-Hai, Israel

4 Emergency Department, Ziv Medical Center, Safed, Israel

5 Management, Ziv Medical Center, Safed, Israel control the extent of the epidemic is to identify the individuals infected with the virus and isolate them from the healthy population (quarantine). Even when a sick person is identified and placed in isolation, the question remains as to the length of the required quarantine.

Several laboratory detection methods are available for COVID-19. These methods have various limitations, including high cost, poor stability, and complex operation process. Some methods require the samples to be collected by professional medical staff [2]. The main methods for diagnosing COVID-19 in Israel are antibody detection, rapid methods for detecting antigens, and polymerase chain reaction (PCR). Although PCR is time-consuming and expensive, it is the gold standard for detection of COVID-19 [3]. This method is based on an enzyme with high sensitivity and strong sequence specificity [4].

The PCR test has become the mainstay in the diagnosis of active disease. Because the PCR test detects portions of viral 
RNA, the test result will continue to be positive long after the virus no longer has contagious capabilities, because only digested and decomposed portions of the virus are excreted from the respiratory airway. One way to verify infectivity is to perform a cell culture for the presence of viable viruses in the sampled secretions. This is a feasible test that takes a long time before an answer is obtained, it is of high cost, not available at most hospitals, and cannot be performed in large numbers like the PCR test, which in Israel can reach up to 200,000 a day.

The basis for the PCR test result is the cycle threshold (CT), which indicates the number of cycles of amplification needed until a positive result is obtained. The lower the response is, the more nucleic acids are in the sample (a larger quantity of viruses). If more and more cycles are needed until a positive result is obtained, it indicates a smaller quantity of viruses.

In practice, the tested individual receives one of the following answers: negative (no tested gene was detected, which usually means a CT above 40), positive (all the genes tested were detected with a CT of up to 40), or borderline positive (depending on the genes detected, and CT values usually ranging between 35 and 40). Medical practitioners and those dealing with pandemics also need to know the $\mathrm{CT}$ value in the positive answer. A great deal of meaning has been attributed to the $\mathrm{CT}$ value, including a negative correlation between the CT level and the risk of deterioration into severe illness and mortality $[5,10]$, and a negative correlation with the degree of infectivity [6, 7]. Subsequent tests can be performed to monitor the dynamics of rising CT values as a sign of a decrease in viral load over time, to determine the possibility of return to work of health-care workers who are infected and in isolation [8], to determine a response to antiviral therapy [9], and more.

In an epidemic, it is important to locate new mutations entering the country at the airport, carried by asymptomatic people or those in the incubation period, to isolate the carriers and prevent the spread of the mutation. It is important to understand that a negative answer can be obtained even when there is a contagious virus in the sample, if the amount of material sampled is very small, or in other words, when the small quantity of viruses resulted in a CT value higher than the limit defined for a "negative answer." This suggests that the quantity of material sampled affects the quantity of virus in the sample, and therefore also the CT value produced by the test. A sample obtained by sustained and intense contact with the mucous membranes and nose of a corona patient contains many more viruses than a sample collected in a short, superficial, and not too deep contact with the mucous membranes.

In none of the studies that discuss the meaning of CT values was the exact method of taking the swab specified. The researchers' assumption was that the standard of sampling is detailed on the CDC website $[11,12]$ under "Interim Guidelines for Collecting and Handling of Clinical Specimens for COVID-19 Testing." A review of the sampling guidelines reveals that they are generic, not sufficiently detailed, and do not guarantee that at different times sampling will be performed in exactly the same way, to obtain uniform samples. Studies comparing CT value dynamics assume that the sampling procedure has no effect on the quantity of viruses in the sample, but that the quantity of viruses depends only on the stage of the disease. Because no study has accurately described the method of sample collection, if our assumption that the method of sample collection affects the quantity of sampled material is correct, there may be a significant bias if a precise and detailed specification of the collection method is not provided.

Furthermore, the quantity of material sampled (assuming that the collection method directly affects the amount of sampled substance) depends on the operation of obtaining the sample. There may be differences between obtaining the samples from a child who opposes and from a cooperating adult. Similarly, if there is a sudden influx of customers (like in the arrivals hall of an airport), the collection procedure may be superficial compared to locations where individuals arrive at a controlled pace, which allows for a higher-quality sampling.

Our research hypothesis is that the way in which the sample is obtained for a PCR test has a significant effect on the resulting CT. Therefore, it is not possible to consider a CT result without a clear and detailed indication of how the sample was obtained, and certainly it is not possible to compare dynamics between results over time without an accurate indication of the sampling method.

\section{Methods}

Approval of the Institutional Ethics Committee (ZIV-20210076) was obtained.

At Ziv Medical Center, PCR tests for COV2 are performed for patients suspected of having the virus upon their arrival to the emergency room (ER).

\section{Inclusion criteria}

1. Clinical complaints consistent with COVID-19 (fever, sore throat, cough, malaise, loss of sense of taste and smell).

2. Referral to the emergency room for any reason, but showing one of the above clinical complaints.

3. Ability to understand and sign a consent form to participate in the study.

4. Age 18 years or older. 


\section{Exclusion criteria}

1. Known to be COVID-19 positive at the time of arrival at the ER present or in the past.

2. Refusal to participate in the study.

For each patient included in the study, two swabs were obtained in sequence. The first swab was obtained using the local procedure (the Ziv procedure) that has been used at the Ziv Medical Center since the beginning of the epidemic. According to the Ziv procedure, the first swab is inserted into the oral cavity, until touching the deep palate; the tester performs three full turns to the right and three full turns to the left. The swab is withdrawn from the pharynx and inserted, parallel to the jaw line, into the right nostril until it comes in contact with the bone. Three full turns to the right and three full turns to the left are performed. A second swab is inserted, using the same method, into the left nostril, and a sample is obtained in the same way. The two swabs are inserted into a sampling tube containing $3 \mathrm{ml}$ of Universal Transport Medium (UTM) transfer buffer.

The second sample was obtained according to the CDC procedure [7], in which a first swab is inserted into the oral cavity touching the palate deep to the right, and the swab is gently rubbed and rolled. Next, the same is performed for the palate deep to the left, and again the swab is gently rubbed and rolled. The swab is withdrawn and inserted into the right nostril parallel to the jaw line until it comes in contact with bone, followed by a gentle rub and roll, after which the swab is removed. A second swab is inserted into the left nostril and a sample is collected in the same manner as from the right nostril. The two swabs are inserted into a sample tube containing UTM.

The samples collected using the $\mathrm{Ziv}$ procedure were marked as Sample 1, and those obtained using the CDC procedure as Sample 2.

After receiving the samples in the laboratory, Sample 1 was inserted into the first sample to result method, the MDX device (Diasorin) according to the protocol of the manufacturer, which identifies the spike (S) and open reading frame (ORF) genes. According to laboratory procedures, each positive sample was verified with an additional device. Therefore, each positive sample was inserted into the second sample to result, the GeneXpert device, which identifies the nucleocapsid $(\mathrm{N})$ and envelope (E) genes according to the protocol of the manufacturer (Cepheid). If a Sample 1 tested positive using both procedures, its corresponding Sample 2 was subjected to the same analysis procedure.

All the positive samples were kept in the laboratory at $80{ }^{\circ} \mathrm{C}$. After collecting all the samples (Samples 1 and 2) and processing them through the sample to result methods, extraction was performed using the Nimbus platform (Hamilton, USA), and then PCR was performed using the
Allplex ${ }^{\mathrm{TM}}$ 2019-nCoV assay (Seegene, South Korea) on the CFX96 (Bio-Rad, USA) in line with manufacturer's instructions. The gene targets for the PCR assay were the E gene (specific of the subgenus Sarbecovirus), the N and S/ RNA-dependent RNA polymerase (RdRp) genes (specifics of the SARS-CoV-2). The Ct value was recorded for each gene. The patients' details, the processing results on all the devices, and the CT values were documented.

\section{Statistical analysis}

For continuous parameters, we used means, medians, standard deviations (SD) and a 95\% confidence interval (95\% CI). Differences between the two types of measurements were determined using the Wilcoxon non-parametric test. A $p$ value of $5 \%$ or less was considered statistically significant. The data were analyzed using SPSS Version 25.

\section{Results}

Over the study period, 100 symptomatic patients were sampled, of whom 20 were found to be positive. Their median age was 38 years (IQR 29-68), and 60\% were men. Median duration of symptoms prior to emergency room arrival was 1.8 days (IQR $1-5$ ) (Table 1 ).

Swabs were obtained using the Ziv method (Sample 1) and the CDC method (Sample 2). All the positive samples were processed by three methods: sample to result molecular methods (MDX, GeneXpert) and Allplex 2019-nCoV assay on the Seegene platform. The CT values of each test were recorded, and a comparison was carried out between the CT results of each gene. In all tests, positive responses $(\mathrm{CT}<40)$ were obtained using both methods.

Statistical results for the CT values of the high-speed tests (Table 2) show that for all four genes tested there are statistically significant differences ( $p$ significantly lower than 0.05 ).

Statistical results for CT values of the PCR tests performed by Allplex 2019-nCoV assay on Seegene platform (Table 3) show significant differences for all three genes with $p=0.001$.

Table 1 Cohort characteristic $(N=20)$

\begin{tabular}{lc}
\hline Characteristic & \\
\hline Age, years, median (IQR) & $38(29-68)$ \\
Gender $(n, \%)$ & $8(40)$ \\
Women & $12(60)$ \\
Men & $1.8(1-5)$ \\
$\begin{array}{l}\text { Days of symptoms prior to obtaining PCR sample, } \\
\text { days, median (IQR) }\end{array}$ \\
\hline
\end{tabular}


Table 2 CT values of sample to result molecular methods PCR COV2 tests

\begin{tabular}{lllllllll}
\hline MDXIGENE & S1 & S2 & ORF1 & ORF2 & E1 & E2 & N1 & N2 \\
\hline Mean & 18.7 & 23.9 & 19.9 & 24.9 & 17.8 & 23.0 & 19.6 & 25.1 \\
Median & 17.1 & 22.0 & 18.9 & 23.8 & 17.1 & 23.4 & 19.3 & 25.4 \\
SD & 4.7 & 5.8 & 4.5 & 5.2 & 2.0 & 2.8 & 2.0 \\
$95 \%$ CI & $15.5-21.8$ & $20.0-27.8$ & $16.9-22.9$ & $21.4-28.4$ & $15.6-19.9$ & $20.0-25.9$ & $17.6-21.7$ & $22.0-28.1$ \\
$p$ value & 0.003 & & 0.003 & & 0.028 & & 0.028 \\
\hline
\end{tabular}

1-Ziv method sampling; $2-$ CDC method sampling; $p<0.05$ considered statistically significant

$S D$ standard deviation

Table 3 CT values of regular (long-extraction) PCR COV2 tests on the Seegene platform

\begin{tabular}{lllllll}
\hline CFX & N1 & N2 & RDRPIS1 & RDRPIS2 & E1 & E2 \\
\hline Mean & 24.8 & 32.8 & 24.1 & 32.2 & 22.8 & 30.6 \\
Median & 23.5 & 31.2 & 22.3 & 30.7 & 21.2 & 29.0 \\
Sd & 5.0 & 5.9 & 5.3 & 6.2 & 5.0 & 6.3 \\
$95 \%$ CI & $21.9-27.7$ & $29.3-36.2$ & $21.1-27.2$ & $28.6-35.7$ & $19.9-25.7$ & $27.0-34.2$ \\
$p$ value & 0.001 & & 0.001 & & 0.001 & \\
\hline
\end{tabular}

1-Ziv method sampling; $2-\mathrm{CDC}$ method sampling; $p<0.05$ considered statistically significant

\section{Discussion}

For nearly 2 years, an epidemic has been raging all over the world on an unprecedented scale. The world is forced to learn and understand facts related to the virus while fighting it and its propagation. The test that has become the gold standard for detecting COV2 and assessing the risk of spreading the disease is the PCR test. The PCR is an indirect quantitative test that provides the result by quantifying the amount of genomic sequence sampled. All over the world, the public receives a binary answer of "positive" or "negative" to make decisions related to isolation. The positive answer has a quantification scale called CT that expresses the number of cycles of augmentation that were required to obtain the positive answer. To the best of our knowledge, the correlation between how the sample had been collected and CT values has never been examined. Because the difference between a positive and negative answer is a range of CT values, a correlation between the method of sampling and the CT response means that the testing results for large populations may change from positive to negative and vice versa. Moreover, there may exist a significant bias in studies that examined CT dynamics over time using different samples without adhering to a standard of sample collection.

This prospective study tested the hypothesis that the way in which the sample is obtained has a decisive effect on the result of the PCR test. In this study, samples were collected from symptomatic patients, and the swabs were sent for testing on two sample to results molecular methods and batch method via the Allplex 2019-nCoV assay (Seegene platform).

The significance of the results obtained with all three devices proves that the method of sample collection has a dramatic effect on results. During an epidemic, when every few months a new contagious variant surges, more dominant than its predecessor, it is important to locate all infected persons as early as possible. Even before the end of the incubation period (2-14 days, 5 days on average after exposure to the COV2 virus), ${ }^{8}$ the secretion of the virus in the respiratory airway begins, with the quantity of virus secreted increasing gradually, until it reaches its peak. Tests performed early, at the onset of virus secretion, will initially produce a negative answer (a high CT value attesting to a low quantity of virus); later the CT values will decrease, attesting to an increase in viral load. A test performed according to the Ziv method yielded a positive response earlier than a test performed using the CDC method. Therefore, a person in the first days of infection, who is asymptomatic, returning from abroad and tested upon arrival, will receive a negative result with the CDC method and a positive one with the Ziv method (probably, with a CT value above 30 ). The difference is significant for the propagation of a new mutant strain brought from abroad, which can be detected with the Ziv method of collection, but not with the CDC method. This can make the difference between preventing or not the spread of a new mutation in the country.

At the airport, upon the arrival of travelers to Israel, PCR tests are performed for everyone entering the country, both those who have been vaccinated and those who have not. Until the result is received, all new arrivals need to be in 
isolation. A negative answer allows a vaccinated or recovering person to leave the quarantine within hours of arriving in the country. A week later, while the virus is still within the incubation period, the unvaccinated can also leave quarantine if their additional PCR result is negative. Even at this point, the person may be in the early stages of secreting the virus (which can last up to two weeks) and obtain a negative answer with the CDC method. By contrast, the Ziv method is much more likely to identify the infection.

With the long lines of travelers waiting for the PCR test at the airport, there is a tendency of the personnel to use the faster method of collecting samples (the CDC method). In the absence of a clear guideline to use a more rigorous sample collection (the Ziv method), the teams carry out the most superficial collection, on the assumption that "what is important is to touch the mucus membrane". Our study demonstrates that all teams performing PCR tests should be instructed to follow a rigorous procedure that requires a deep penetration and contact with mucous membranes, including several rotations of the swab in both directions.

As noted, in many studies, CT values are considered to be in correlation with viral load, so that subsequent tests showing increased CT values express a decrease in viral load, and vice versa. Our study demonstrates that in the absence of an accurate description of sample collection in the research methods, test results cannot be compared, because deeper or more superficial collection may cause significant differences in CT values.

We suggest requiring that publications that report CT values, especially sequential values, state clearly and in detail, in the research methods section, the exact method of sample collection. We recommend using the Ziv method.

The limitations of this research are the small sample, consisting of 20 participants and the higher sensitivity of "Ziv" method we have achieved. The study, which was designed to include 100 participants with positive PCR results, was discontinued because of the high significance of the intermediate results. Continuation of the study could have delayed the implementation of its recommendations and increased the risk of under-diagnosis and of incorrect kinetic information regarding PCR tests. Additionally, Better sensitivity is good for airport screening, but could lead to false positives if positivity is defined as contagious infection: detection of late and non-contagious infection, especially in asymptomatic individuals, would lead e.g. to non-necessary isolation.

\section{Conclusions}

The method of collection (superficial or deep and intensive) has a significant and critical effect on the CT values of PCR tests for coronavirus detection. In places where it is important not to miss individuals in the initial stages of disease (airports, hospitals, classrooms, etc.), it should be required that the testing personnel use the Ziv standard. In research, each CT score should be accompanied by information about the method of collection used in the study, making sure that all the samples were taken in the exact same manner. We recommend using a method such as Ziv, which entails deep and intensive collection.

Conflicts of interest All authors declare that there are no any potential conflicts of interest.

\section{References}

1. https://covid19.who.int

2. Bu J, Deng Z, Liu H, Li J, Wang De, Yang Y, Shian Z. Current methods and prospects of coronavirus detection. Talanta. 2021;225: 121977. https://doi.org/10.1016/j.talanta.2020.121977.

3. Shen M, Zhou Y, Ye J, Abdullah Al-Maskri AA, Kang Y, Zeng S, Cai S. Recent advances and perspectives of nucleic acid detection for coronavirus. J Pharm Anal. 2020;10:97-101.

4. Narushima R, Shimazaki T, Takahashi T. Development of a realtime reverse-transcription-PCR method for detection of RD114 virus in canine vaccines. Biologicals. 2011;39:89-93.

5. Rao SN, Manissero D, Steele VR, Pareja J. A systematic review of the clinical utility of cycle threshold values in the context of COVID-19. Infect Dis Ther. 2020;9:573-86.

6. La Scola B, Le Bideau M, Andreani J, et al. Viral RNA load as determined by cell culture as a management tool for discharge of SARS-CoV-2 patients from infectious disease wards. Eur J Clin Microbiol Infect Dis. 2020;39:1059-61.

7. Bullard J, Dust K, Funk D, et al. Predicting Infectious Severe Acute Respiratory Syndrome Coronavirus 2 From Diagnostic Samples. Clin Infect Dis. 2020;71(10):2663-2666.

8. Domeracki S, Clapp RN, et al. Cycle threshold to test positivity in COVID-19 for return to work clearance in health care workers. J Occup Environ Med. 2020;62:889-91.

9. Chaccour C, Ruiz-Castillo P, et al. The SARS-CoV-2 ivermectin navarra-isglobal trial (SAINT) to evaluate the potential of ivermectin to reduce COVID-19 transmission in low risk, non-severe COVID-19 patients in the first 48 hours after symptoms onset: a structured summary of a study protocol for a randomized control pilot trial. Trials. 2020;21:498.

10. Asai N, Sakanashi D, et al. Could threshold cycle value correctly reflect the severity of novel coronavirus disease 2019 (COVID19)? J Infect Chemother. 2021;27:117-9.

11. https://www.cdc.gov/coronavirus/2019-nCoV/lab/guidelines-clini cal-specimens.html.

12. https://www.who.int/docs/default-source/coronaviruse/situationreports/20200127-sitrep-7-2019--ncov.pdf. 